\title{
Subtyping of Non-Small Cell Lung Carcinoma in Fine Needle Aspiration Specimens: A Study of 252 Patients with Surgical Correlations
}

\author{
Beyhan Varol Mollamehmetoğlu ${ }^{1}$, Gülgün Sade Koçak ${ }^{1}$, Havva Erdem², \\ Ylldıray Bekar ${ }^{3}$
}

${ }^{1}$ Department of Pathology, Kanuni Training and Research Hospital, Trabzon, Turkey

${ }^{2}$ Department of Pathology, Ordu University School of Medicine, Ordu, Turkey

${ }^{3}$ Department of Thoracic Surgery, Kanuni Training and Research Hospital, Trabzon, Turkey

\begin{abstract}
Objective: Fine-needle aspiration (FNA) cytology performed by either transthoracic or transbronchial procedures is an important approach to obtain tumor tissue for histological diagnosis. We investigated the accuracy of FNA in differentiating NSCLCs of adenocarcinoma from squamous cell carcinoma histological types to correlate cytological findings with histological features and immunohistochemistry confirmation in some cases.
\end{abstract}

Methods: From 2010 to 2015, a total of 635 transbronchial needle aspirations or transthoracic needle aspirations were performed. 332 cases were diagnosed as NSCLC, with or without an indication of a specific subtype, while 303 cases were not diagnosed as NSCLC. Out of 332 cases diagnosed as NSCLC, 252 had a histological follow-up. Subsequently, histological samples included 161 surgical resections and 91 biopsies. In cases with histopathological diagnosis accompanied by FNA cytology, an immunohistochemical study was carried out and the diagnostic results of the two methods were compared to each other.

Results: The specific subtype of NSCLC was provided in 217 cases (86\%) based on cytomorphology which included 115 adenocarcinomas $(46 \%)$ and 102 squamous cell carcinomas (40\%). The diagnosis NSCLC-NOS by FNA was set in 35 cases. At histology, 251 cases (99.6\%) were sub-classified: 122 adenocarcinomas (48\%), 104 squamous cell carcinomas (41\%), 11 large cell carcinomas (4\%), and 14 adenosquamous carcinomas (6\%). Agreement between cytological and histological typing was found in 181 of 197 cases $(92 \%)(\mathrm{K}=0.837 ; \mathrm{p}<0.001)$.

Conclusion: Our study proved that most NSCLC can be sub-classified as adenocarcinoma or squamous cell carcinoma by FNA through cytomorphology and the application of immunocytochemistry.

Keywords: Fine-needle aspiration, histological correlation, non-small cell lung cancer

Received Date: 21.03.2016 Accepted Date: 12.06 .2016 Available Online Date: 05.09 .2016

DOI: $10.5152 /$ ejp.2016.15238

Correponding Author

Beyhan Varol Mollamehmetoglu

E-mail: bmollamehmetoglu@yahoo.com

- Available online at www.eurasianjpulmonol.com

This work is licensed under a Creative Commons Attribution-NonCommercial 4.0 International License.

\section{INTRODUCTION}

Lung cancer is the largest cause of adult cancer-related deaths in men and women worldwide (1). With over 1.3 million mortalities per year due to this disorder. In the past, lung cancers were classified into two groups: as small-cell neuroendocrine carcinoma and non-small cell lung cancer (NSCLC). Nonsmall cell lung cancer, with the introduction of new treatment modalities, recognition and differential diagnosis between adenocarcinoma and squamous cell carcinoma is necessary for effective molecular target-specific treatments $(2,3)$. Targets include epidermal growth factor receptors (EGFR), tyrosine kinase inhibitors (TKIs), and echinoderm microtubule associated protein-like 4-anaplastic lymphoma kinase (EML4-ALK) fusion protein (4).

Fine-needle aspiration (FNA) cytology, carried out by either transthoracic or transbronchial techniques, is an important approach to obtain tissue for pathologic diagnosis and ancillary molecular testing such as Epidermal Growth Factor Receptor mutation (EGFR), Anaplastic Lymphoma Kinase (ALK), Kirsten rat sarcoma viral oncogene homolog (KRAS) mutation analyses (4-6). It has been demonstrated in the literature that FNA is simpler, more cost effective, a less invasive way of diagnosing malignancy, and associated with fewer complications than core needle biopsy and open biopsy. Small 
cell lung cancer and NSCLC can be consistently established by FNA, while its role in accurately typing NSCLC tumors is still under debate (7). Advanced treatment procedures, including targeted biological therapies and specific chemotherapeutic agents, define the necessity for undisputed accurate diagnoses with the correct use of immunohistochemical stains and supreme protection of representative tissue.

The present study was performed to determine the diagnostic accuracy of FNA in differentiating NSCLCs of squamous from nonsquamous subtypes, to correlate cytological findings with histological features, and immunohistochemistry confirmation in some cases.

\section{METHODS}

\section{Case Selection}

Cytological reports of FNA for lung lesions carried out in our hospital were reviewed from the digital archives of the Department of Pathology. From 2010 to 2015, a total of 635 transthoracic needle aspirations or transbronchial needle aspirations were performed: 332 cases were diagnosed as NSCLC, with or without a definition of a specific subtype, while 303 cases were not diagnosed as NSCLC (72 negative for tumor, 40 non-diagnostic, 51 suspicious of malignancy, 105 small cell lung cancers, 20 metastatic tumors, and 6 lymphomas). Data regarding additional relevant histopathological diagnosis were reviewed for the study: Results of transbronchial forceps, core needle biopsies, and resections (wedge, lobectomy, and pneumonectomy).

\section{Cytological Diagnosis}

Peripheral lung masses were sampled thorough transcutaneous computed tomography (CT)-guided FNA by a thoracic surgeon. FNA central lung masses, as well as hilar or mediastinal lymph nodes, were sampled through endobronchial ultrasound guidance by a pulmonologist. Transbronchial needle aspiration was performed for lesions in the vicinity of large bronchi or mediastinal lymph node sampling. Transbronchial needle aspiration was performed with a Wang 21-gauge cytology needle. CT-guided transthoracic needle aspirations were performed in patients with pulmonary masses using a 22-gauge disposable spinal needle attached to a $10 \mathrm{ml}$ syringe to obtain cytological material. The number of needles passed depended on the sufficiency of the cytological sample gained. Air-dried and wet-fixed (95\% alcohol) cytology smears were constructed and stained by May-Grunwald-Giemsa (MGG) and Papanicolaou stain or hematoxylin-eosin (H\&E) stain. Additional passes were requested for the cell block if ancillary studies were necessary. In 87 cases, adequate cell preparations were available.

\section{Histological Diagnosis}

Subsequently, histological samples involved 161 surgical resections and 91 biopsies. 22 of 91 biopsies were core needle biopsies. All the tumor slides stained with H\&E and periodic acid-Schiff (PAS) stains. Two experienced pathologists subtyped the resected samples on the basis of the H\&E slides considering the World Health Organization classification of lung tumors. Discrepant cases were resolved with the aid of immunostains.

Immunocytochemical stains were routinely performed on adequate cell block samples. The antibodies used in the study were TTF-1 (thyroid transcription factor-1), Napsin A, CK5/6 (cytokeratin 5/6), and p63. The detection system used was a Leica Bondmax autostainer (Leica, Buffalo, IL, USA). CK5/6 and/or p63 positive cases were considered consistent with squamous cell carcinoma, while TTF-1 and/or Napsin A staining favored adenocarcinoma classification.

\section{Statistical Analysis}

Statistical Package for the Social Sciences version 20.0 (SPSS IBM Statistics, Armonk, New York, USA) was used for all analyses. The sensitivity, specificity, diagnostic accuracy, and negative and positive predictive values of FNA were calculated. Kappa statistics was used to estimate the level of agreement. Kappa values ranging from 0.81 to 1 were assumed to indicate an almost perfect agreement. $P$ value $<0.05$ was considered significant.

\section{Ethics}

The ethics committee of the Kanuni Training and Research Hospital approved this study. As a retrospective study, the ethical committee does not ask for written informed consent from the patients.

\section{RESULTS}

In the six year period from 2010 through 2015, 635 FNA for diagnoses of lung lesions were carried out at our training hospital. An algorithmic approach showing the summary of the study is seen in Figure 1. The average age of patients was 63.4 years (range 25-89). There were 392 males and 243 females $(\mathrm{M} / \mathrm{F}$ ratio $=1.6)$. Malignant lesions were diagnosed in 463 FNA (73\%). The type and relative frequency of malignant tumors is seen in Figure 2. Out of 332 cases diagnosed as NSCLC, 252 had a histological follow-up. 252 FNA cases (201 transthoracic and 51 transbronchial) having subsequent histological diagnosis were included into the study. Biopsies represented the correlating surgical specimens with 91 of the 252 follow-up specimens being core or forceps biopsies that were obtained either concomitantly or within 2 months. Follow-up resection specimens (segmentectomy, lobectomy, or pneumonectomy) were noted in 161 cases.

The specific subtype of NSCLC was provided in 217 cases (86\%) based on cytomorphology, immunocytochemistry, and clinical history with comparisons of archived histological and cytological materials in our files. This included 115 adenocarcinomas (46\%) and 102 squamous

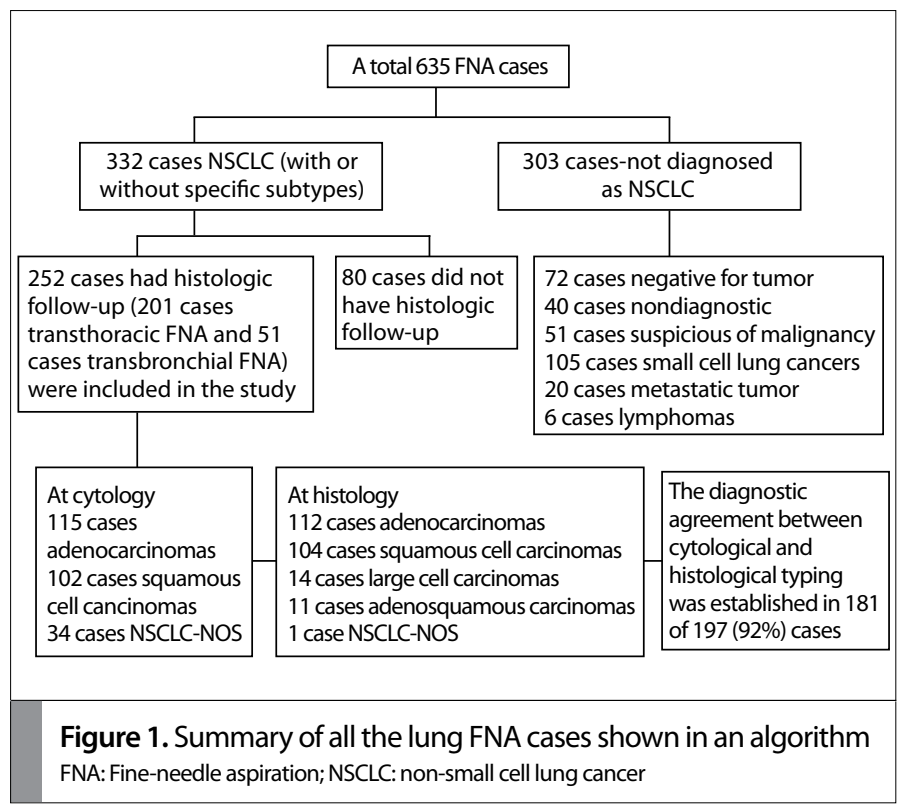




\begin{tabular}{|l|c|c|}
\hline \multirow{2}{*}{ Table 1. Distributions of NSCLC subtypings } \\
\cline { 2 - 3 } & \multicolumn{2}{|c|}{ Diagnosis } \\
\hline $\begin{array}{l}\text { Adenocarcinoma } \\
\text { (or NSCLC-favor ADC) }\end{array}$ & $115(53)$ & $122(49)$ \\
\hline $\begin{array}{l}\text { squamous cell carcinoma } \\
\text { (or NSCLC favor SqCC) }\end{array}$ & $102(47)$ & $104(41)$ \\
$\begin{array}{l}\text { Large cell carcinoma } \\
\text { (or NSCLC favor LCC) }\end{array}$ & $11(4)$ \\
$\begin{array}{l}\text { Adenosquamous carcinoma } \\
\text { (or NSCLC favor ADSqC) }\end{array}$ & - & $14(6)$ \\
\hline $\begin{array}{l}\text { Total } \\
\text { NSCLC-NOS }\end{array}$ & 217 (86) \\
$\begin{array}{l}\text { A total of 251 cases had cytological and histological subtype diagnosis of } \\
\text { NSCLC. NSCLC-NOS: Non-small cell lung cancer not otherwise specified }\end{array}$ \\
\hline
\end{tabular}

Table 2. Cytological diagnosis in 252 FNA with corresponding histopathological diagnosis

\begin{tabular}{|c|c|c|c|c|c|c|}
\hline $\begin{array}{l}\text { Cytology/ } \\
\text { Histopathology }\end{array}$ & $\begin{array}{c}\mathrm{SqCC} \\
\text { (n) }\end{array}$ & $\begin{array}{c}A D C \\
(n)\end{array}$ & $\begin{array}{r}\text { LCC } \\
\text { (n) }\end{array}$ & $\begin{array}{c}\text { ADSqC } \\
\text { (n) }\end{array}$ & $\begin{array}{c}\text { NOS } \\
(n)\end{array}$ & $\begin{array}{c}\text { Total } \\
\text { (n) }\end{array}$ \\
\hline SqCC & 87 & 10 & 3 & 2 & - & 102 \\
\hline ADC & 6 & 94 & 6 & 9 & - & 115 \\
\hline NOS & 11 & 18 & 2 & 3 & 1 & 35 \\
\hline Total & 104 & 122 & 11 & 14 & 1 & 252 \\
\hline
\end{tabular}

Table 3. Comparison between FNA and histopathological type (SqCC vs ADC)

\begin{tabular}{|l|c|c|c|}
\hline Cytology/Histopathology $(\mathbf{n})$ & ADC & SqCC & Total \\
\hline ADC & 94 & 6 & 100 \\
SqCC & 10 & 87 & 97 \\
Total & 104 & 93 & 197 \\
\hline
\end{tabular}

ADC: Adenocarcinoma; SqCC: squamous cell carcinoma

cell carcinomas (40\%). NSCLC-NOS by FNA was diagnosed in 35 cases (14\%) where no apparent glandular squamous differentiation was represented by either cytomorphologically or immunocytochemical methods (Table 1). Negative immunohistochemical stains for cytokeratin $5 / 6$, cytokeratin 7 , TTF 1 , and p63 were shown in 11 cases that had cell blocks. The material was inadequate for immunohistochemical stains in 13 cases. Immunocytochemical stains were performed in 87 cases that had cell blocks (Figure 3 ).

At histology, 251 cases were sub-classified (99.6\%): 122 adenocarcinomas (48\%), 104 squamous cell carcinomas (41\%), 11 large cell carcinomas (4\%), and 14 adenosquamous carcinomas (6\%) (Table 2). Immunostains were performed in 64 biopsy specimens (70\%) and in 93 resection specimens (58\%). Only one case at forceps biopsy (1\%) was considered as NSCLC-NOS owing to scanty material or tumor necrosis.
Cytological and histological results were compared to acquire agreement rates. The comparison between cytological and histological matching was possible in 197 cases. Except for 35 cases reported as NSCLC-NOS, 9 cases were diagnosed as large cell carcinoma, and 11 cases diagnosed as adenosquamous cell carcinoma at histology (Table 2).

The diagnostic agreement between cytological and histological typing was established in 181 of 197 cases (92\%) ( $K=0.837 ; \mathrm{p}<0.001)$. Compatible diagnoses involved 94 adenocarcinomas and 87 squamous cell carcinomas (Table 3). 10 cases diagnosed as squamous cell carcinoma at cytology were classified as adenocarcinoma after resection specimens while 6 cases were diagnosed as adenocarcinoma after cytology followed squamous cell carcinoma at histology. Of the 35 NSCLC-NOS cases at cytology, histological typing was achieved in 34 cases with the aid of immunohistochemistry.

In our study, histopathological \pm immunostaining reports of NSCLC were considered as final confirmatory reports. Positive predictive values of FNA in NSCLC typing were $90 \%$ (87 of 97) for squamous cell carcinoma and $94 \%$ (94 of 100) for adenocarcinomas. Sensitivity, specificity, positive predictive values of FNA, and negative predictive values of FNA for a diagnosis of adenocarcinomas were $90 \%, 94 \%$, $94 \%$, and $90 \%$, respectively.

\section{DISCUSSION}

The results of this study show that FNA allows sub-classification of NSCLCs with high accuracy, according to the agreement with histology (92\%), and the positive predictive value for squamous and nonsquamous subtypes ( $90 \%$ and $94 \%$, respectively).

The distinction of NSCLC subgroups is very important in proper management as the EGFR-directed TKIs, erlotinib and gefitinib, are effective in patients with adenocarcinoma having the EGFR mutation, while patient with squamous cell carcinoma should not be given bevacizumab because of the risk of life-threatening pulmonary hemorrhage. Accurately sub-categorizing NSCLCs into adenocarcinoma and squamous cell carcinoma influences clinical decision making and drives the appropriate treatment selection. Current literature on the topic supports cytological subtyping of NSCLCs is feasible and accurate, especially when cytomorphologic studies are combined with immunocytochemistry and cytochemical staining (5-8). However, some authors have suggested that FNA is not useful in sub-classifying NSCLCs due to the difficulty of assessing poorly differentiated tumors, the limited nature of the specimens, and a decreased ability to assess architectural patterns compared to histology (9).

These subtypes are usually easily distinguished by FNA when they are well differentiated. The prevalent technical factors related with misdiagnosis were poor spread, poor fixation, weak cellularity, and hemorrhage. When cellularity was found to be sufficient in the absence of any technical wrongdoing, the patterns of different lesions and cell sizes were confused with each other. In most situations, more than one factor was determinant. Reproducible cytomorphologic differences between squamous cell carcinoma and adenocarcinoma have been described. In adenocarcinoma, cells are usually found in 3-dimensional arrangements with prominent nucleoli, finely vacuolated cytoplasm, and indistinct cell borders. In contrast, tumor cells of squamous cell carcinoma have sharp cell margins, dense cytoplasm, nuclear hyperchromasia, and remarkable nuclear 
membrane irregularities. Spindle cell morphology and "tadpole" cells are common in conventional Papanicolaou stains, and are helpful by highlighting keratinizing cells, followed by modified Giemsa stained

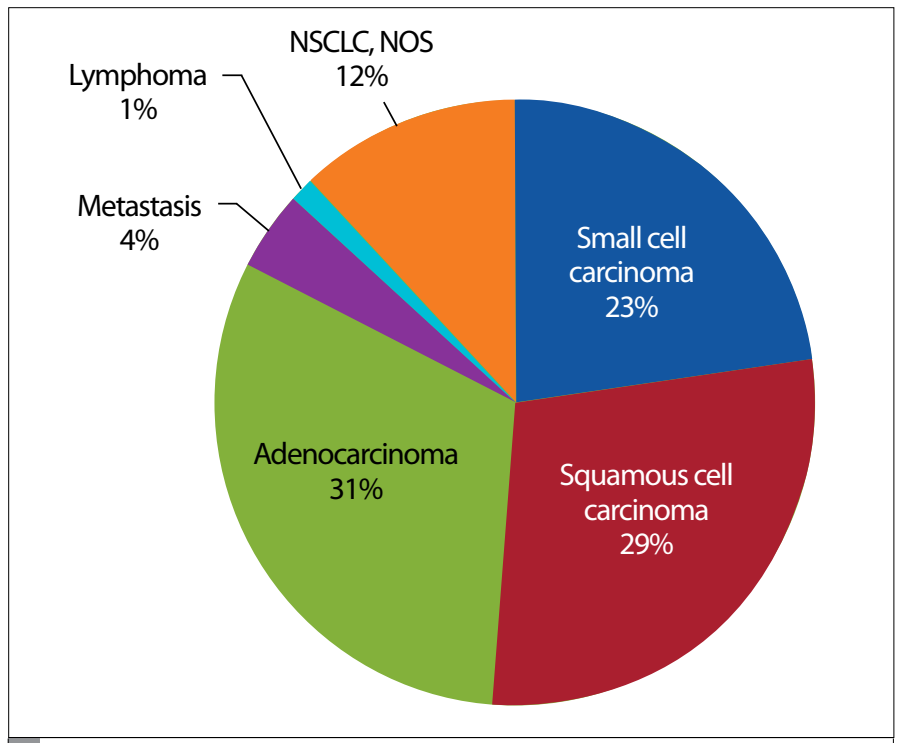

Figure 2. Type and relative frequency of 463 malignant lung lesions NSCLC: Non-small cell lung cancer and liquid-based preparations (5). This superior ability is supported by Sigel et al. (10) who state that morphologic examination is better in cytology than surgical pathology with the use of different stains helping to achieve a morphological classification. Rekhtman et al. (11), in a study of 192 cytology diagnoses, when compared with resection specimens, found the accuracy of cytological diagnosis was $93 \%$. When IHC was used in $9 \%$ the cases, the accuracy was $100 \%$. Nizzoli et al. (7) examined 1182 transbronchial/transthoracic FNA samples, 474 of which had a cytologic diagnosis of primary NSCLC. At cytology, $85 \%$ NSCLC cases were typed while $15 \%$ were classified as NSCLC-NOS. Cytologic and histologic typing was compatible in $88 \%$ of the cases. The positive predictive value of FNA in typing NSCLC was $82 \%$ for squamous cell carcinoma and $92 \%$ for adenocarcinoma.

While light microscopic evaluation of cytomorphological features remains the crucial first step for sub-classifying NSCLCs, poorly differentiated non-small cell carcinomas may lack characteristic features. In these instances, in our study, immunohistochemical stains performed on the cell block allowed for appropriate sub-classification of difficult cases. The 2011 IASLC/ATS/ERS lung adenocarcinoma classification suggests using only one adenocarcinoma marker (Napsin A or TTF-1) and a single squamous marker (p63 or CK5/6) for NSCLC classification in small biopsy or cytology specimens in the absence of certain glandular or squamous morphologies to reserve tissue for future molecular testing (12). We were able to successfully perform

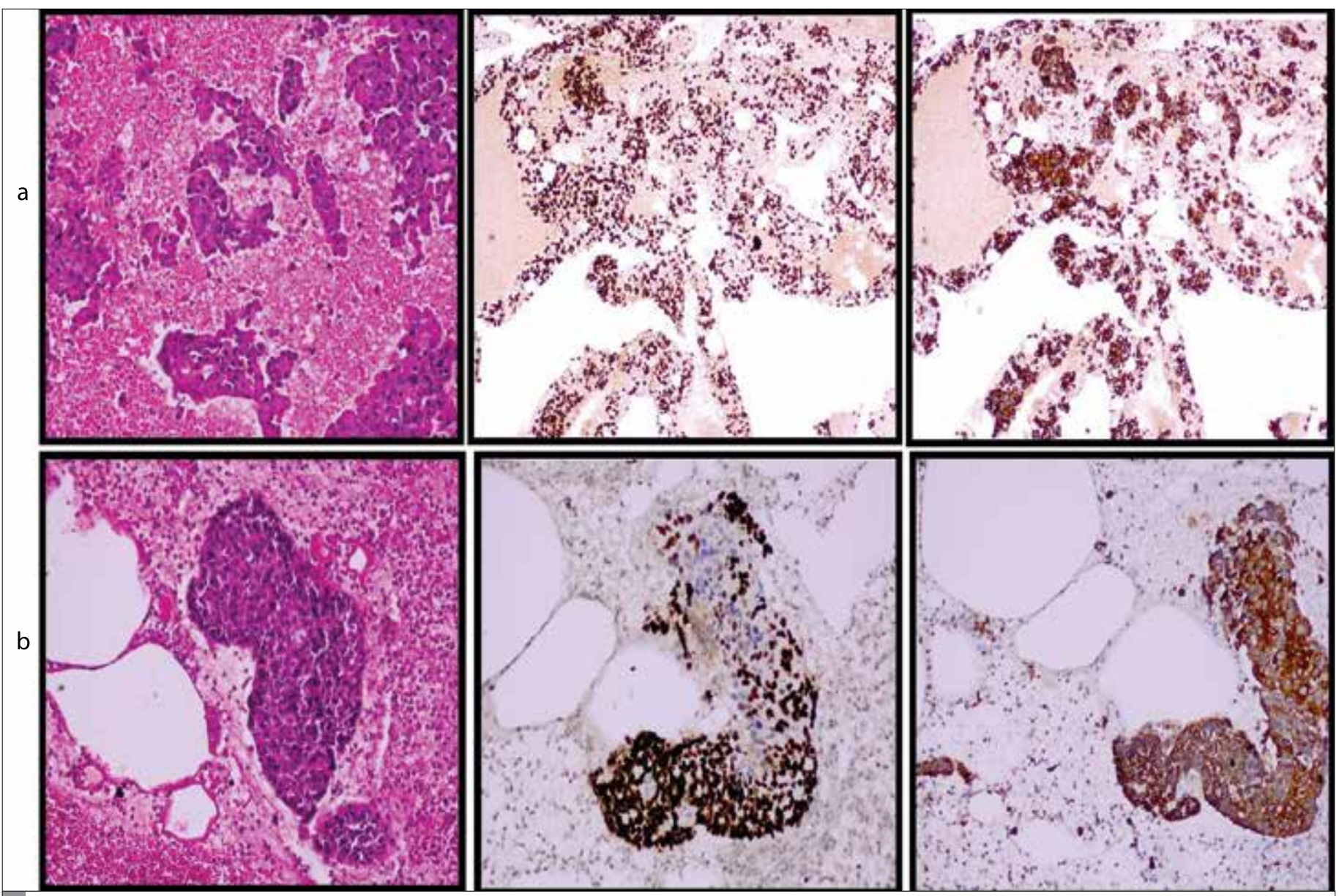

Figure 3. a, b. (a) Adenocarcinoma. Left, cytomorphology of ADC on cell block slide (H\&E); center, immunostain of TTF-1 in tumor cells; right, immunostain of Napsin A in tumor cells. (b) Squamous cell carcinoma. Left, cytomorphology of SqCC on cell block slide (H\&E); center, immunostain of p63 in tumor cells; right, immunostain of CK5/6 in tumor cells 
immunohistochemical stains in 87 cases (35\%) of FNA specimens. Of the 252 cases of NSCLC in our study, we were able to subtype $86 \%$ cases and specifically diagnose adenocarcinoma in 115 cases and squamous cell carcinoma in 102 cases. Khayyata et al. (13) assessed the role of cytomorphology and added the benefit of immunocytochemistry in differentiating adenocarcinoma from squamous cell carcinoma in 53 lung FNA specimens. Using only cytomorphologic criteria, concordance rates for adenocarcinoma and squamous cell carcinoma were $66 \%$ and $53 \%$, respectively (combined accuracy $60 \%)$, but were increased when immunohistochemistry was involved in the diagnostic algorithm. In contrast, Sigel et al. (10) reported that the morphological sign of differentiation as adenocarcinoma or squamous cell carcinoma is more obvious in cytological specimens supported by less frequent utilization of immunocytochemistry for subtyping in cytology than in small biopsy specimens (32\% versus $6 \%, p<.001$ ). Adams et al. (1), in a study of 1032 patients determining the accuracy of FNA for the diagnosis of lung cancer, reported that FNA had a $97 \%$ diagnostic accuracy for malignant tumors and a $100 \%$ specificity. Gong et al. (14) demonstrated that, for malignant tumors, the diagnostic accuracy of FNA was comparable to that of core needle biopsy, with an accuracy of $85.1 \%$ and $86.7 \%$, respectively.

Shibuki et al. (15) described that TTF-1 and Napsin A were useful for improving the diagnostic accuracy of lung squamous cell carcinoma and adenocarcinoma with cytological samples. P63 has generally been applied as a marker of squamous cell carcinoma, and is considered to be a beneficial and highly sensitive marker for routine histologic diagnosis. Additionally, its use is beneficial with cytological specimens because of its expression in the nucleus. Immunomarkers against $\mathrm{CK} 5 / 6$ and $\mathrm{CK} 5$, which display no reaction in the vast majority of adenocarcinomas, have recently been used for the detection of high-molecular weight CK. In this study, the four antibodies were able to segregate even poorly differentiated cases of lung carcinoma using cell blocks made from FNA specimens.

With recent advancements in targeted therapy for patients with advanced NSCLC, it is important for material collected by FNA to be able to be used for molecular studies to identify EGFR, BRAF, KRAS, MET, PIK3CA, and ROS1 mutations in adenocarcinoma and PIK3CA and p16 in squamous cell carcinoma, as targeted therapies have had promising results. Molecular testing for these alterations has typically been obtained by core-needle biopsy or surgical resection because of the belief that cytology material is insufficient for molecular testing. However, cytology specimens have been shown to be sufficient for molecular testing. Importantly, the use of cytology specimens for molecular testing prevents patient exposure to more invasive surgical procedures with a higher morbidity (16-19).

This study was a retrospective study obtained from the hospital registry for analysis. A large scale prospective or case controlled type study will be more reliable to establish a statistical significance and correlation.

\section{CONCLUSION}

Our study proved that most NSCLC can be sub-classified as adenocarcinoma or squamous cell carcinoma by FNA through cytomorphology and the application of immunocytochemistry. We evaluated the most commonly used four IHC markers, including TTF-1, Napsin A, p63, and CK5/6, in the sub-classification of NSCLC. We found that these immunopanels were effective for sub-classifying NSCLC into adenocarcinoma and squamous cell carcinoma. A further prospective study using an independently collected cohort is necessary to validate our results.

Ethics Committee Approval: Ethics committee approval was received for this study from the ethics committee of Kanuni Sultan Süleyman Training and Research Hospital.

Informed Consent: As retrospective study, the ethical committee does not require the written informed consent from the patients.

Peer-review: Externally peer-reviewed.

Author Contributions: Concept - B.V.M., G.K.; Design - B.V.M.; Supervision B.V.M., H.E.; Resources - B.V.M., Y.B.; Materials - B.V.M., G.K., Y.B.; Data Collection and/or Processing - B.V.M., H.E., G.K.; Analysis and/or Interpretation - B.V.M., H.E.; Literature Search - B.V.M., H.E.; Writing Manuscript - B.V.M.; Critical Review - B.V.M., H.E.; Other - G.K., Y.B.

Conflict of Interest: No conflict of interest was declared by the authors.

Financial Disclosure: The authors declared that this study has received no financial support.

\section{REFERENCES}

1. Adams J, Wu HH. The utility of fine-needle aspiration in the diagnosis of primary and metastatic tumors to the lung: a retrospective examination of 1,032 cases. Acta Cytologica 2012; 56:590-5. [CrossRef]

2. Ikeda S, Naruse K, Nagata C, Kuramochi M, Onuki T, Inagaki M, et al. Immunostaining for tyroid transcription factor $1, N a p \sin A, p 40$, and cytokeratin 5 aids in differential diagnosis of non-small cell lung carcinoma. Oncol Lett 2015; 5: 2099-104.

3. Kapila K, Al-Ayadhy B, Francis IM, George SS, Al-Jassar A. Subclassification of pulmonary non-small cell lung carcinoma in fine needle aspirates using a limited immunohistochemistry panel. J Cytol 2013; 30: 223-5. [CrossRef]

4. Gurda GT, Zhang L, Wang Y, Chen L, Geddes S, Cho WC, et al. Utility of five commonly used immunohistochemical markers TTF-1, Napsin A, CK 7, CK5/6 and P63 in primary and metastatic adenocarcinoma and squamous cell carcinoma of the lung: a retrospective study of 246 fine needle aspiration cases. Clin Transl Med 2015; 4: 16. [CrossRef]

5. Yildiz-Aktas IZ, Sturgis CD, Barkan GA, Souers RJ, Fraig MM, Laucirica R, et al. Primary pulmonary non-small cell carcinoma. The collage of American pathologists interlaboratory comparison program confirms a significant trend toward subcategorization based upon fine-needle aspiration cytomorphology alone. Arch Pathol Lab Med 2014; 138: 65-70. [CrossRef]

6. Sethi S, Geng L, Shidham VB, Archuletta P, Bandyophadhyay S, Feng J, et al. Dual color multiplex TTF-1 + Napsin A and p63 + CK7 immunostaining for subcategorizing of poorly differentiated pulmonary non-small carcinomas into adenocarcinoma and squamous cell carcinoma in fine needle aspiration specimens. CytoJournal 2012; 9: 10. [CrossRef]

7. Nizzoli R, Tiseo M, Gelsomino F, Bartolotti M, Majori M, De Filippa M, et al. Accuracy of fine needle aspiration cytology in the pathological typing of non-small cell lung cancer. J Thorac Oncol 2011; 6: 489-93. [CrossRef]

8. Khorsandi M, Shaikhrezai K, Wallance W, Brackenbury E. Is fine-needle diagnosis of malignancy adequate prior to major lung resections including pneumonectomy? Interact Cardiovasc Thorac Surg 2012; 15: 253-7. [CrossRef]

9. Langer CJ, Besse B, Gualberto A, Brambilla E, Soria JC. The evolving role of histology in the management of advanced non-small-cell lung cancer. J Clin Oncol 2010; 28: 5311-20. [CrossRef]

10. Sigel CS, Moreira A, Travis WD, Zakowski MF, Thomton RH, Riely GJ, et al. Subtyping of non-small cell lung carcinoma: a comparison of small biopsy and cytology specimens. J Thorac Oncol 2011; 6: 1849-56. [CrossRef]

11. Rekhtman N, Brandt SM, Sigel CS, Friedlander MA, Riely GJ, Travis WD, et al. Suitability of thoracic cytology for new therapeutic paradigms in non-small cell lung carcinoma: high accuracy of tumor subtyping and 
feasibility of EGFR and KRAS molecular testing. J Thorac Oncol 2011; 6: 451-8. [CrossRef]

12. Travis WD, Brambilla E, Nouguchi M, Nicholson AG, Geisinger KR, Yatabe $Y$, et al. International association for the study of lung cancer/american thoracic society/european respiratory society international multidisciplinary classification of lung adenocarcinoma. J Thorac Oncol 2011; 6: 244-85. [CrossRef]

13. Khayyata S, Yun S, Pasha T, Jian B, McGrath C, Yu G, et al. Value of P63 and CK5/6 in distinguishing squamous cell carcinoma from adenocarcinoma in lung fine-needle aspiration specimens. Diagn Cytopathol 2009; 37: 178-83. [CrossRef]

14. Gong Y, Sneige N, Guo M, Hicks ME, Moran CA. Transthoracic fine-needle aspiration vs. concurrent core needle biopsy in diagnosis of intrathoracic lesions: a retrospective comparison of diagnostic accuracy. Am J Clin Pathol 2006; 125: 438-44. [CrossRef]
15. Shibuki Y, Tsuta K, Nomoto K, Maezawa N, Tochigi N, Maeshima A, et al. Immunocytochemical study of specific immunohistochemical markers for primary lung adenocarcinoma: Surfactant apoprotein A, Narsin A, Thyroid transcription factor-1. J Jpn Clin Cytol 2006; 45: 6-11. [CrossRef]

16. Sharma SV, Settleman J. ErbBs in lung cancer. Exp Cell Res 2009; 315: 557-71. [CrossRef]

17. Billah S, Stewart J, Staerkel G, Chen S, Gong Y, Guo M. EGFR and KRAS mutations in lung carcinoma: molecular testing by using cytology specimens. Cancer Cytopathol 2011; 119: 111-7. [CrossRef]

18. Desai A, Menan SP, Dy GK. Alterations in genes other than EGFR/ALK/ ROS1 in non-small cell lung cancer: trials and treatment options. Cancer Biol Med 2016; 13: 77-86. [CrossRef]

19. van Eijk R, Licht J, Schrumpf M, Talebian Yazdi M, Ruano D, Forte Gl, et al. Rapid KRAS, EGFR, BRAF, and PIK3CA mutation analysis of fine needle aspirates from non-small-cell lung cancer using allele-specific Qpcr. PLoS One 2011; 6: e177-91. [CrossRef] 